\section{Positive impact of infection prevention on the management of nosocomial outbreaks at an academic hospital}

\author{
Jan-Willem H Dik', Bhanu Sinha', Mariëtte Lokate', Jerome R Lo-Ten-Foe', \\ Ariane G Dinkelacker ${ }^{1,2}$, Maarten J Postma ${ }^{3,4,5}$ \& Alexander W Friedrich ${ }^{*, 1}$
}

\begin{abstract}
Aim: Infection prevention (IP) measures are vital to prevent (nosocomial) outbreaks. Financial evaluations of these are scarce. An incremental cost analysis for an academic IP unit was performed. Material \& methods: On a yearly basis, we evaluated: IP measures; costs thereof; numbers of patients at risk for causing nosocomial outbreaks; predicted outbreak patients; and actual outbreak patients. Results: IP costs rose on average yearly with $€ 150,000$; however, more IP actions were undertaken. Numbers of patients colonized with high-risk microorganisms increased. The trend of actual outbreak patients remained stable. Predicted prevented outbreak patients saved costs, leading to a positive return on investment of 1.94. Conclusion: This study shows that investments in IP can prevent outbreak cases, thereby saving enough money to earn back these investments.
\end{abstract}

First draft submitted: 15 February 2016; Accepted for publication: 1 April 2016; Published online: 11 April 2016

The unwanted spread of microorganisms within healthcare institutions can lead to major problems, such as nosocomial infections and outbreaks, resulting in increased morbidity and mortality [1,2]. Besides these highly undesirable clinical effects, these problems also cost considerable amounts of finances and resources. [1,3-4]. The worldwide problem of antimicrobial resistance further increases the risk of difficult or even impossible to treat nosocomial outbreaks with multi-drug-resistant organisms (MDROs). It is therefore vital to have a proactive infection prevention department or infection control program that actively screens and acts upon possible outbreak risks. Actions by these departments, such as hand hygiene measures or the search-and-destroy policy for MRSA, have already demonstrated positive clinical results that can be achieved [5-7]. All these measures do, however, come at a price. Present-day studies on clinical effects of infection prevention and/or control are still in need of improvement [8,9]. Consequently, proper economic evaluations of these interventions, keeping in mind all potential biases that are inherent to this field, are also scarce [10]. It is most likely that infection prevention measures can be cost effective and can yield substantial return on their investment [4]. However, some measures will be more cost effective than others, achieving the same clinical goals. Having the financial information on these measures will improve the decision-making processes.

\footnotetext{
'Department of Medical Microbiology, University of Groningen, University Medical Center Groningen, Hanzeplein 1, 9713GZ, Groningen, The Netherlands

2Department of Medical Microbiology, University Hospital Tübingen, Elfriede-Aulhorn-Straße 6, 72076, Tübingen, Germany ${ }^{3}$ Department of Pharmacy, Unit of PharmacoEpidemiology \& PharmacoEconomics, University of Groningen, Antonius Deusinglaan 1, 9713AV, Groningen, The Netherlands

${ }^{4}$ Institute of Science in Healthy Aging \& healthcaRE (SHARE), University Medical Center Groningen, Hanzeplein 1, 9713GZ, Groningen,

The Netherlands

${ }^{5}$ Department of Epidemiology, University Medical Center Groningen, Hanzeplein 1, 9713GZ, Groningen, The Netherlands

*Author for correspondence: Tel.: +31 50 3613480; alex.friedrich@umcg.nl
}

\section{KEYWORDS}

- cost-benefit analysis

- health economics

- infection prevention

- nosocomial outbreaks
Future $\%$ (1) 
This study sets out to evaluate the Infection Prevention Unit and its budget at an academic hospital over eight subsequent years. Because of the inherent difficulties in evaluating costs occurring due to healthcare associated infections mentioned before, we focus purely on the costs of nosocomial outbreaks, the prevention thereof and its impact on the whole infection prevention budget, through an incremental cost-benefit analysis. Each year, the infection prevention budget has been increased to cope with the rise in antimicrobial resistance. This study evaluates the yearly incremental rise of the budget to calculate a yearly return on investment (ROI). Such an approach eliminates the problems of having to estimate a baseline situation without infection control measures based on numerous highly uncertain assumptions. This study should be seen as a first step toward calculating a proper and comprehensive financial analysis that incorporates all costs and benefits of infection prevention aspects.

\section{Materials \& methods}

The data within this study concern eight subsequent years, from 2007 up to 2014, at a tertiary academic center in the north of The Netherlands. During this period, national infection prevention protocols changed due to updates, however, the general approaches remained similar [11]. General hospital data, such as number of admissions per year, average length of stay (LOS) and number of beds per year, were taken from the hospital's annual reports [12]. Microbiological culture data were collected from the database of the Department of Medical Microbiology. Data of consumables were based on the purchase details from the hospital's purchasing department. For the cost analysis of the outbreaks, data came from a previous cost analysis, done within the same hospital on a subset of the outbreaks investigated here [13]. A hospital perspective was used with 2012 price levels.

\section{- Determining the size of the Infection Prevention Unit}

For each year, the total number of infection prevention specialists employed by the hospital in December of the respective year was taken in full-time equivalent (FTE; based on $36 \mathrm{~h}$ per week). This number of FTEs was further increased by $10 \%$ of the total FTE of clinical microbiologists to account for their pro rata infection prevention work, and for additional related researchers and technicians in the field of infection prevention, including the next-generation sequencing group of the department. The number of nursing staff for the whole hospital was also evaluated over the same time period to control if possible changes was due to a hospital-wide trend or an independent effect.

\section{- Evaluating different (indirect) infection prevention quality indicators}

To evaluate the effect of the Infection Prevention Unit, different (indirect) quality indicators were chosen that were objectively recorded over the years and that were available for data analysis. Four quality indicators were used: the total amount of hand disinfection alcohol; the number of surveillance cultures performed; the total amount of disposables; the results of point-prevalence studies for adherence to the dress codes. Disposables taken into account were: nonsterile gloves; coats; caps; aprons; and mouth-nose masks. All disposables are used mainly or exclusively for infection prevention and control measures as stated in the hospital's local guidelines. Surveillance cultures were defined as the following swabs: nose, nose/throat, nose/ perineum, nose/throat/perineum, throat/rectum, and rectum. These were done in general for MRSA risk patients as defined by the Dutch MRSA guideline [14] and for certain other (resistant) microorganisms depending on the ward and/or patient. The point-prevalence studies were hospital-wide studies performed five-times in 2012 and 2013. For each department it was scored how the personnel adhered to the dress code guidelines. Six parameters were scored: no nail polish; correct hair-do; no jewellery; closed coats; no long sleeves (protruding from under the short sleeves from white coats); and correct outfit (long white coat or short white coat plus white trousers).

\section{- Incidence of risk microorganisms}

The incidence per 1000 admissions of colonization of nine different (multidrug resistant) microorganisms, for which the propensity for nosocomial outbreaks is known, was evaluated. We choose to look at the resistant strains of the so-called ESKAPE organisms plus two extra local additions: MRSA; extended-spectrum $\beta$-lactamase (ESBL) and/or carbapenemaseproducing Klebsiella pneumonia; multi-resistant Pseudomonas aeruginosa; vancomycin-resistant Enterococcus faecium and Enterococcus faecalis (VRE); Serratia marcescens; Acinetobacter 
baumanii; and Norovirus (requiring a positive PCR and complaints). All positive cultures in the microbiological database were evaluated over the respective 8 years. Enterobacter spp. culture results were not available completely for the whole 8 years and could therefore not be included. Duplicate isolates for individual patients were removed.

\section{- Determining the predicted \& observed colonized patients during nosocomial outbreaks}

For the evaluated time period all outbreaks at the hospital as defined as such by the Infection Prevention Unit were evaluated. An outbreak was defined as spread (i.e., colonization) of the same strain of a microorganism (confirmed by either genotyping methods [e.g., MLVA, spa typing] or whole-genome sequencing from 2012 on) among patients or personnel, with an epidemiological link between the positive cases (e.g., same ward, same room), within a specific time-frame (depending on the type of microorganism). All outbreaks were handled at the time of occurrence by the Infection Prevention Department and total numbers of positive patients were counted for all outbreaks.

Based on the number of risk microorganisms we calculated the incremental (yearly) rise/drop in terms of percentage and used these to calculate the predicted (yearly) rise/drop for the number of outbreak patients, giving a (yearly) predicted number of outbreak patients which can be compared with the actual (yearly) found number of outbreak patients. Considering the possible effect of the average LOS in the hospital on the incidence, the total number of predicted outbreak patients has been corrected for a change in LOS.

\section{- Calculating a ROI}

Using the data from nosocomial outbreaks at the same hospital, an average duration per colonized patient and price per colonized patient per outbreak day was calculated. A cost analysis was performed for seven outbreaks with pathogenic microorganisms (e.g., MRSA, ESBL $K$. pneumoniae and VRE) that occurred between 2012 and 2014 (the included seven are, therefore, also part of all investigated outbreaks in this study). The calculated median cost per patient per outbreak day over the seven different outbreaks in 2012 price level was $€ 499$ and the weighted mean duration was 36.9 days [13]. To account for the variation within this cost analysis, we took the medians instead of the average to be on the conservative side. These data were used for the rest of the financial evaluation.

ROI has been calculated for each year by taking the total amount of yearly investments into infection prevention and dividing those by the yearly incremental costs or benefits. This yielded a yearly ratio that represents the amount of costs or benefits for each Euro invested. To calculate the ROI, the total budget of the Infection Prevention Unit was determined at $€ 1.5$ million for 2012 . Due to the expected rise in risks and resistance, the budget has been increased every year due to proactive investments into infection prevention. Infection prevention is an integral part of the Department of Medical Microbiology within this hospital and budgets are combined, making yearly detailed discrimination of costs (e.g., overhead costs) not always possible. Therefore, an estimated budgetary increase was made of $€ 100,000$ per year, taking into account the increased number of personnel. This is most likely an overestimation, which implies that our incremental costbenefit approach can be considered conservative. Furthermore, the incremental change in costs of the consumables used for infection prevention on the wards was added to the respective yearly investments.

\section{- Statistics \& calculations}

To analyze the trends over the years and calculate if there was a significant rise, decrease or no change, we performed univariate linear regression analyses for each single variable against time (8 years). Because numbers can fluctuate during years, we chose to evaluate a period of eight subsequent years to level out potential outliers. To analyze a correlating effect between the number of surveillance cultures and microorganisms found, binary logistic regression analyses were performed. To correct for a possible ascertainment bias on the incidence of the microorganisms in relation with the average LOS of the hospital, positive patients were analyzed. Day of first positive culture was scored (thus taking into account the moment of positivity) and a formula was plotted to calculate the cumulative incidence over time. For each year the average LOS in the hospital was compared with the first year (2007) and with the formula the difference in cumulative incidence was calculated and subtracted from the total percentage. A univariate sensitivity analysis was performed to evaluate the impact 
of some of the parameters. A single parameter was varied $\pm 25 \%$ with the rest of the parameters at their baseline level. A significance level of $\mathrm{p} \leq 0.05$ was applied. All calculations were done with Microsoft Excel (Microsoft, WA, USA) and SPSS (IBM, NY, USA).

\section{Results}

\section{- The hospital had a growing Infection Prevention Unit}

For the hospital, the total amount of infection prevention personnel was inventoried per year. The number includes infection prevention specialists, clinicians and supporting personnel (e.g., researchers). From 2007 to 2014 the unit saw a $65 \%$ increase $(p=0.025)$. The total number of nursing staff per hospital bed remained stable (average of $46.03 \mathrm{FTE} / 1000 \mathrm{admissions),}$ with no significant change $(\mathrm{p}=0.167)$. The average LOS showed a statistically significant downward trend $(\mathrm{p}<0.01)$ (see Table 1).

\section{- Coincidently infection prevention policy} quality indicators improved

To evaluate the impact of the increased infection prevention staff on adherence to infection prevention protocols within the hospital, four quality indicators were measured: the number of surveillance cultures; the amount of hand disinfection alcohol; use of disposables (i.e., gloves, coats, caps, aprons and masks); and the adherence to the dress codes. All quality indicators showed a significant change over time (Figure 1A-C \& Table 1). Compared to 2007, in 2014 use of hand alcohol went up by $43 \%$, surveillance cultures by $131 \%$ and total amount of disposables by $69 \%$ (only the use of caps decreased, the rest of the disposable increased in use). The adherence to the dress codes was measured five times in a hospital-wide pointprevalence study in the last 2 years and adherence went up by $25 \%$. Six factors were looked at: no nail polish; correct hair-do; no jewellery; closed

\begin{tabular}{|c|c|c|c|c|c|c|c|c|c|c|}
\hline Outcome measures & 2007 & 2008 & 2009 & 2010 & 2011 & 2012 & 2013 & 2014 & $\Delta$ 2007-2014 ${ }^{\dagger}$ & p-value \\
\hline Hospital beds (n) & 1339 & 1339 & 1339 & 1339 & 1339 & 1339 & 1339 & 1339 & $0 \%$ & $\mathrm{~N} / \mathrm{A}$ \\
\hline Admissions ( $\mathrm{n}$ ) & 32,831 & 34,411 & 35,412 & 35,842 & 36,892 & 36,695 & 37,249 & 34,671 & $6 \%$ & $p=0.10$ \\
\hline Average LOS (days) & 9.94 & 9.58 & 9.18 & 8.96 & 8.71 & 8.34 & 8.21 & 8.87 & $-11 \%$ & $\mathrm{p}<0.01$ \\
\hline Nursing personnel $(n)^{\ddagger}$ & 46.08 & 45.76 & 44.64 & 44.99 & 43.94 & 45.76 & 46.44 & 50.68 & $10 \%$ & $p=0.17$ \\
\hline IP personnel $(n)^{\ddagger}$ & 0.25 & 0.26 & 0.23 & 0.25 & 0.26 & 0.28 & 0.31 & 0.42 & $65 \%$ & $p=0.03$ \\
\hline \multicolumn{11}{|l|}{ IP quality indicators ${ }^{\ddagger}$} \\
\hline Hand alcohol (I) & 287 & 277 & 288 & 288 & 312 & 329 & 361 & 410 & $43 \%$ & $p=0.02$ \\
\hline Gloves (n) & 1835 & 1872 & 2036 & 2144 & 2513 & 2603 & 2802 & 4214 & $130 \%$ & $\mathrm{p}<0.01$ \\
\hline Coats $(n)^{\S}$ & - & - & 4520 & 5595 & 6749 & 6549 & 7599 & 8140 & $80 \%$ & $\mathrm{p}<0.01$ \\
\hline Cap (n) & 3911 & 3982 & 2909 & 2741 & 2953 & 2327 & 2493 & 2909 & $-26 \%$ & $p=0.03$ \\
\hline Aprons (n) & 5744 & 6048 & 6676 & 6796 & 6562 & 8170 & 7928 & 10055 & $75 \%$ & $\mathrm{p}<0.01$ \\
\hline Masks (n) & 4975 & 4653 & 4909 & 4581 & 4993 & 5136 & 5973 & 6608 & $33 \%$ & $\mathrm{p}<0.01$ \\
\hline Surveillance cultures (n) & 214 & 193 & 270 & 308 & 288 & 329 & 367 & 498 & $132 \%$ & $p=0.01$ \\
\hline Dress code adherence & - & - & - & - & - & - & $62 \%$ & $79 \%$ & - & N/A \\
\hline \multicolumn{11}{|l|}{ Incidence risk organisms ${ }^{\ddagger}$} \\
\hline Staphylococcus aureus (MRSA) & 1.25 & 1.28 & 0.99 & 1.06 & 1.19 & 1.50 & 1.42 & 1.73 & $39 \%$ & $\mathrm{~N} / \mathrm{A}$ \\
\hline Klebsiella pneumonia (ESBL and KPC) & 0.15 & 0.61 & 1.47 & 1.53 & 2.33 & 2.67 & 2.07 & 1.85 & $1112 \%$ & $\mathrm{~N} / \mathrm{A}$ \\
\hline Pseudomonas aeruginosa (MDR)\# & 0.22 & 0.53 & 0.47 & 0.46 & 0.68 & 0.67 & 0.66 & 0.57 & $157 \%$ & $\mathrm{~N} / \mathrm{A}$ \\
\hline Enterococcus faecium/Enterococcus faecalis (VRE) & 0.00 & 0.00 & 1.33 & 3.12 & 2.60 & 0.79 & 0.72 & 2.34 & $\infty$ & $\mathrm{N} / \mathrm{A}$ \\
\hline Serratia marcescens & 3.54 & 3.61 & 3.19 & 3.75 & 3.16 & 3.86 & 3.02 & 3.14 & $-11 \%$ & $\mathrm{~N} / \mathrm{A}$ \\
\hline Acinetobacter baumanii & 0.00 & 0.03 & 0.03 & 0.00 & 0.00 & 0.00 & 0.00 & 0.03 & $\infty$ & $\mathrm{N} / \mathrm{A}$ \\
\hline Norovirus & 2.98 & 3.07 & 2.88 & 4.38 & 4.01 & 3.13 & 4.75 & 4.41 & $48 \%$ & $\mathrm{~N} / \mathrm{A}$ \\
\hline Predicted outbreak patients ${ }^{\ddagger}$ & 1.55 & 1.71 & 1.89 & 2.40 & 2.32 & 2.05 & 2.05 & 2.30 & $48 \%$ & $\mathrm{~N} / \mathrm{A}$ \\
\hline Observed outbreak patients ${ }^{\ddagger}$ & 1.55 & 0.26 & 0.96 & 4.02 & 0.95 & 1.12 & 1.96 & 0.95 & $-39 \%$ & $\mathrm{~N} / \mathrm{A}$ \\
\hline \multicolumn{11}{|c|}{$\begin{array}{l}\text { 'Difference between first and last year. } \\
\text { FPer } 1000 \text { admissions. } \\
\text { SDue to unavailability of data from } 2007 \text { and 2008, difference presented here is between } 2009 \text { and } 2014 . \\
\text { "Average percentage per year based on multiple point-prevalence audits. } \\
\text { "MDR is resistance for at least three of the following: ceftazidime, meropenem, ciprofloxacin, piperacillin-tazobactam, gentamicin. } \\
\text { ESBL: Extended spectrum beta-lactamase; KPC: Klebsiella producing carbapenamase; LOS: Length of stay; MDR: Multiple drug resistant; N/A: Not applicable; VRE: Vancomycin- } \\
\text { resistant Enterococcus. }\end{array}$} \\
\hline
\end{tabular}




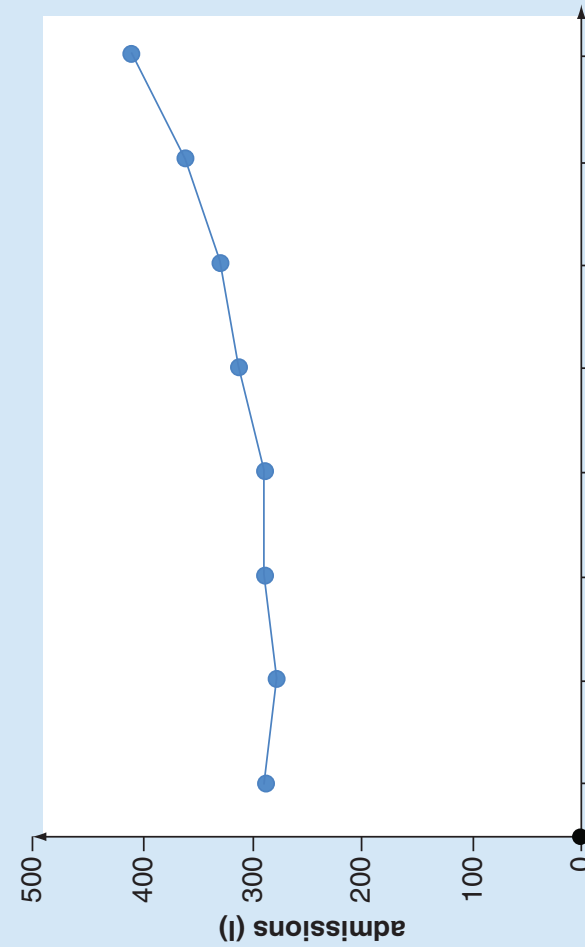

(a) 000L//очоगе uо!|әәјu!s!p pueH
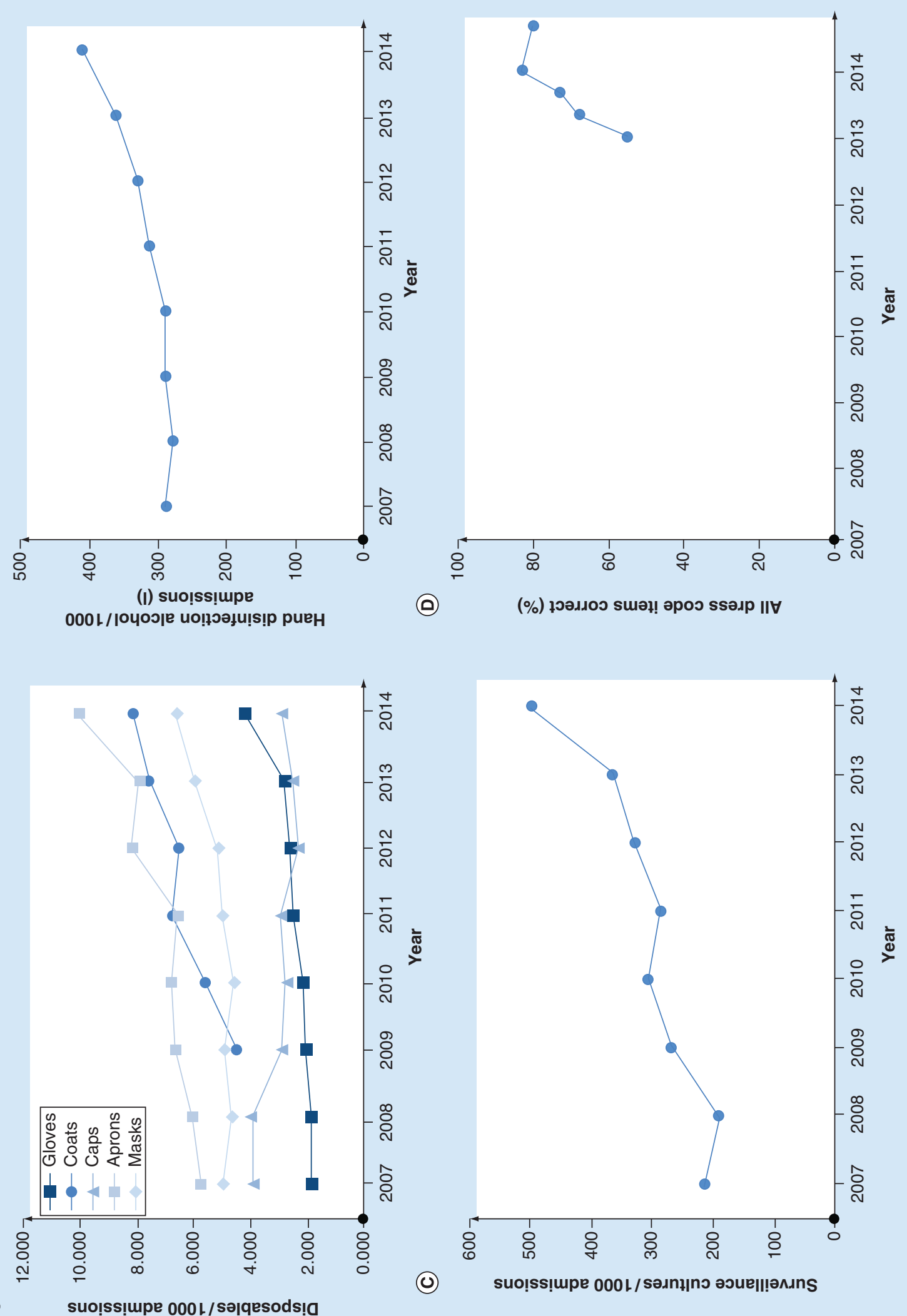

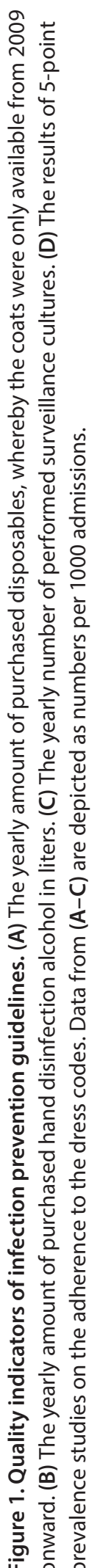


coats; no long sleeves (protruding from under the short sleeves from white coats); and correct coat (Figure 1D \& Table 1).

\section{- The hospital faced an increase in risk microorganisms}

All cultures within the evaluated period of 8 years were taken into account and positive cultures were counted for risk microorganisms, whereby duplicate isolates from individual patients were excluded. Over the 8 years, there was an increased incidence for all microorganisms within the hospital (Figure 2 \& Table 1). Only $P$. aeruginosa and $S$. marcescens were found to correlate significantly with the total number of surveillance cultures $(\mathrm{p}=0.007$ and $\mathrm{p}=0.037$, respectively). The total number of positive cultures was corrected for this correlation. Considering the significant reduction in average LOS in the hospital that was seen over the eight years, the total number of risk microorganisms was corrected for this drop.

\section{- The number of involved patients during nosocomial outbreaks remained at a stable level}

For all nosocomial outbreaks within the hospital, the number of colonized patients were counted and evaluated. The trend remained constant over the eight evaluated years in contrast to the yearly incremental increase in risk microorganisms (see Table 1).

\section{- This difference in expected positive patients \& identified positive outbreak patients prevented substantial costs}

The incremental yearly difference in risk microorganisms over the last 8 years was used to predict the number of outbreak patients and compared with the actual observed outbreak patients. Using the previously calculated median cost of one extra outbreak day per positive patient (€499) [13], the number of prevented incremental outbreak patients was quantified in a monetary value, giving a yearly value of incremental costs or savings (Table 2). On average this gave a yearly ROI of 1.94 (median: 3.87 ) ranging from -8.74 to 6.77 (Table 2). The univariate sensitivity analysis showed, with one exception, only positive average yearly ROIs, ranging from -0.41 to 4.28 (Figure 3).

\section{Discussion \& conclusion}

This study investigated the Infection Prevention Unit of a Dutch academic hospital retrospectively during an 8-year period from 2007 to 2014. During these years the number of resistant microorganisms rose within the hospital, most likely due to increased admission of patients carrying MDRO. This trend is seen in The Netherlands as well in Europe and the rest of the world [15]. This pressure is further increased by a high connectivity between hospitals, with academic centers (such as this one) often acting as a central hub [16]. Partly as a response to this rising pressure, there was a significant rise in infection prevention personnel. Coincidently with this rise, several (indirect) quality parameters for infection prevention protocols also saw a rise. We hypothesize that more infection prevention personnel leads to increased awareness and better adherence to the different guidelines. Proving a direct correlation is difficult. However, the rise in the eight different quality parameters does show undoubtedly that more protocol actions are performed. The question remains if they were performed correctly. During the 8 years there was also a considerable drop in the observed outbreak patients when comparing them with the predicted numbers. Also here, proving a direct correlation is difficult, but by looking at multiple parameters and correcting for several confounders, we tried to minimize the risk of a biased conclusion. The observed drop in outbreak patients can be quantified financially. In an earlier study, looking at seven different outbreaks (using the same definition as in this study) in the same hospital, a weighted mean duration of an outbreak and a median price per patient per day was calculated [13]. Using these data, this study shows, as one of the first, the positive incremental bundle effect of an Infection Prevention Unit at an academic hospital. Nosocomial outbreak patients were prevented and the yearly extra investments that were done to keep up with the rise in highrisk microorganisms as well as the rise in antimicrobial resistance levels had an overall positive return on investment (ROI). This was evaluated over a period of eight subsequent years, to rule out large influences of potential positive or negative outliers (i.e., years).

The Netherlands has a good infection prevention track record. High screening rates and the proactive search-and-destroy policy for MRSA are good examples of this [5]. In total there are 78 different national infection prevention protocols [11] that are keeping prevalence of risk microorganisms low. Even so, the rise in resistant microorganisms worldwide is reflected 


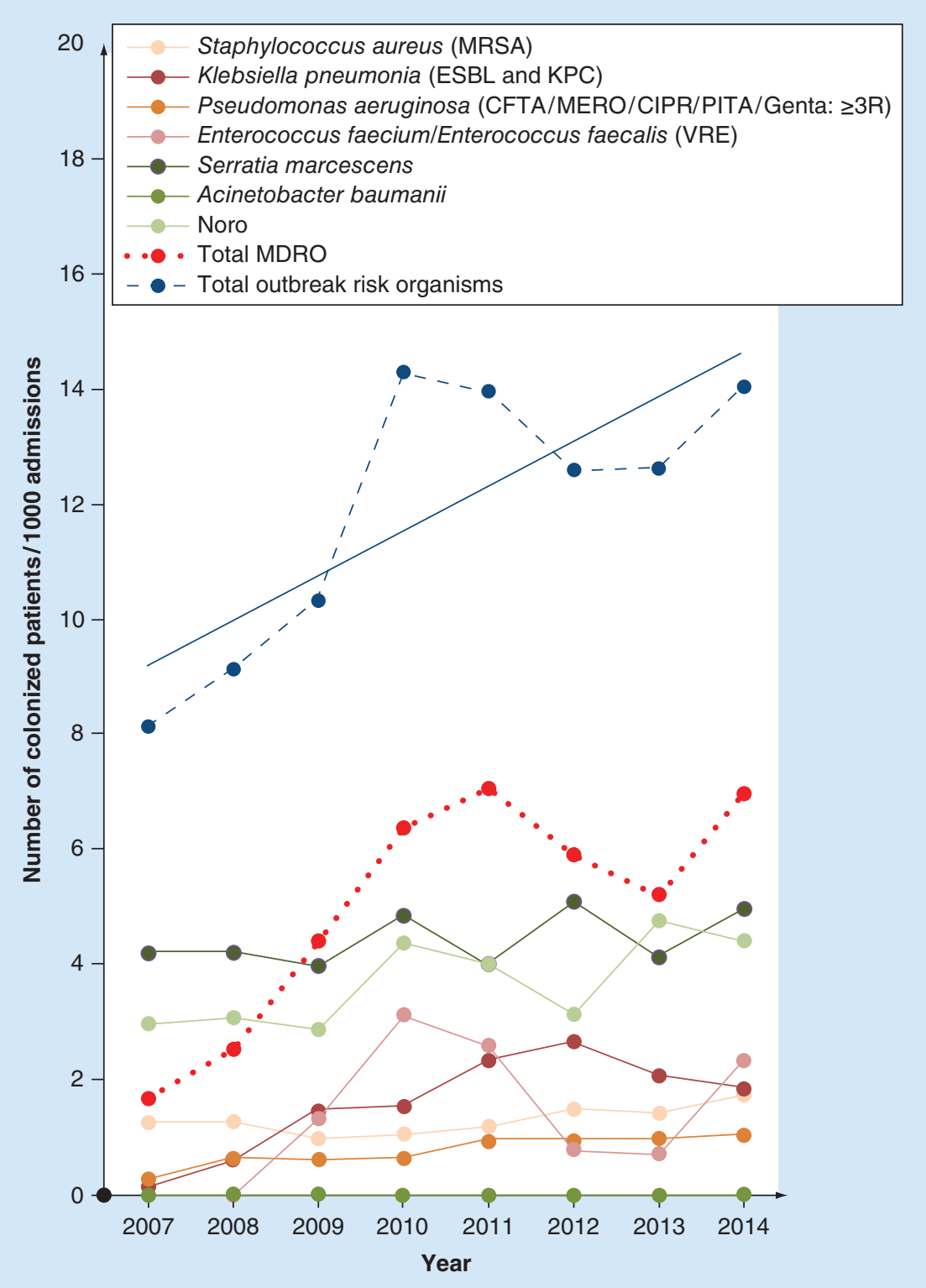

Figure 2. Incidence of risk microorganisms. Yearly incidence from 2007 until 2014 of the risk microorganisms as defined within the graph. Data are plotted as colonized patients per 1000 admissions. Most upper barred blue line represents the total amount with the linear trend line; the red dotted line represents the total amount of multi drug resistant organisms. NB: colonized in the case of norovirus means having a positive PCR and complaints; the peak in 2010 is caused by a VRE outbreak, which was considered unrepresentatively large.

CFTA: Ceftazidime; CIPR: Ciprofloxacin; ESBL: Extended spectrum $\beta$-lactamase; Genta: Gentamicin; KPC: Klebsiella producing carbapenamase; MDRO: Multi-drug-resistant organism; MERO: Meropenem; PITA: Piperacillin-tazobactam; VRE: Vancomycin-resistant Enterococcus.

in the Dutch situation as well [15]. It is therefore important to anticipate for higher prevalence of MDROs by investing in infection prevention. For this hospital, the present number of staff per bed seems to be sufficient for a low incidence country and the rise in infection prevention specialists seems to be able to keep up with the rise in MDROs. Although outbreaks did occur more frequently, the number of positive patients per outbreak dropped, possibly indicating that outbreaks are more quickly contained (data not shown). This appears to be case nationwide in 


\begin{tabular}{|c|c|c|c|c|c|c|c|}
\hline Year & $\begin{array}{l}\text { Predicted } \\
\text { outbreak patient } \\
\text { days }\end{array}$ & $\begin{array}{l}\text { Observed } \\
\text { average patient } \\
\text { days }\end{array}$ & $\begin{array}{l}\text { Difference in } \\
\text { outbreak patient } \\
\text { days }\end{array}$ & $\begin{array}{l}\text { Difference } \\
\text { outbreaks costs } \\
\text { (€) }\end{array}$ & $\begin{array}{l}\text { Incremental rise } \\
\text { infection } \\
\text { prevention } \\
\text { budget }(€)\end{array}$ & $\begin{array}{l}\text { Difference } \\
\text { infection } \\
\text { prevention costs } \\
(€)\end{array}$ & ROI \\
\hline 2007 & 1872 & 1872 & - & - & - & - & - \\
\hline 2008 & 2157 & 330 & 1826 & 958,912 & 134,049 & 782,341 & 5.84 \\
\hline 2009 & 2455 & 1248 & 1207 & 633,520 & 195,515 & 409,912 & 2.10 \\
\hline 2010 & 3154 & 5285 & -2131 & $-1,118,736$ & 138,130 & $-1,207,257$ & -8.74 \\
\hline 2011 & 3147 & 1285 & 1862 & 977,749 & 191,843 & 742,549 & 3.87 \\
\hline 2012 & 2763 & 1505 & 1258 & 660,660 & 115,491 & 515,873 & 4.47 \\
\hline 2013 & 2799 & 2679 & 120 & 62,756 & 222,046 & $-162,072$ & -0.73 \\
\hline 2014 & 2923 & 1211 & 1712 & 898,711 & 110,526 & 748,333 & 6.77 \\
\hline
\end{tabular}

The Netherlands [17] and corroborates that the (national and regional) approach to infection prevention is the main contributor. Of course, these results can only be obtained by having the proper facilities and staff in a healthcare institution [18] and proper harmonized infection control educational programs between healthcare institutions [19].

Up to 2007, the Dutch national norm for hospitals was 1 FTE of infection prevention specialist per 250 beds (5.4 FTE for this hospital) [20]. This changed to 1 FTE per 5000 admission from 2007 onward (6.6 FTE for this hospital) [21]. This is comparable to Australia [22], and slightly lower than US data [23]. The updated Dutch norm in 2012 did not increase number of personnel, but focused more on quality of their work and correct implementation of different guidelines [24-26]. This hospital employed even more personnel (from 2011 on), although the definition of infection prevention personnel used in this study is somewhat broader (including supporting staff (e.g., mathematical modelers) as well; but no laboratory technicians and medical specialists mainly active in diagnostic and/or antimicrobial stewardship). Besides infection prevention personnel, it is known that the number of nurses per hospital bed and their workload also influences patient safety and infection rates [27-29]. A change in the nurse per admission ratio could thus be a confounding factor. It was therefore taken into

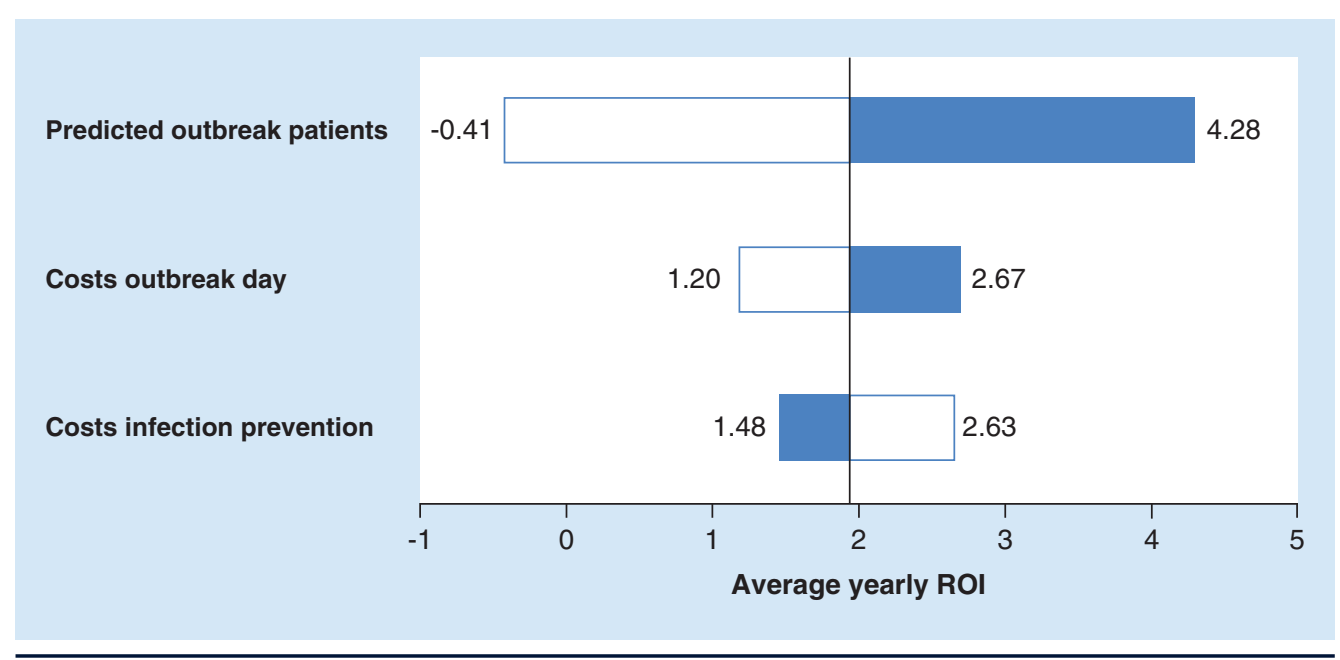

Figure 3. Univariate sensitivity analysis. Three parameters that used assumptions were varied with $25 \%$ and the average yearly $\mathrm{ROI}$ is shown centered around the average yearly ROI found with the baseline values (1.94). The assumed $€ 100,000$ yearly increase in infection prevention costs, the $€ 499$ costs of one outbreak day per patient and the number of predicted outbreak patients were varied. Nearly all of them gave positive average yearly ROIs. Colored bars are $+25 \%$, open bars $-25 \%$. ROI: Return on investment. 
account in this study. There was a stable rate over the 8 years and National Dutch numbers for academic hospitals showed similar stable trends [30]. Infection rates most likely increase when LOS increases, a so-called ascertainment bias if not taken into account. Therefore also this factor was evaluated. Average LOS in the hospital showed significant drop over the years, most likely due to other improvements in healthcare and logistics (although this was not examined in detail as it was not part of the study) and the predicted outbreak patient number was consequently corrected for this drop. Indirect measurement of infection prevention through the amount of hand alcohol or disposables (product volume measurement [PVM]) is done before [31,32]. It is a relatively easy method and does not require labor intensive audits. It is unfortunately not ideal because it does not provide information on the way these consumables are used. However, audits are not always an option, certainly not when analyzing data retrospectively. We therefore feel that by evaluating not just hand alcohol, but a set of parameters, that all showed similar trends, the causal relation we hypothesize is plausible. Finally, to account for the possible effect that a change in assumed parameters has, a sensitivity analysis was performed.

We show that savings can be achieved by investing in the prevention of outbreaks and thus preventing specific measures or actions such as closed wards (reducing the revenue opportunities) and usage of personnel (creating extra costs by hiring temporary staff or opportunity costs by redistributing existing staff). Savings mentioned in this study are based upon the previous cost analysis of outbreaks within the same hospital. Depending on the outbreak, different savings will be achieved, but in general it can be expected that savings are more or less similar distributed as within the cited study (i.e., $50 \%$ is saved on prevention of bed/ward closure, $17 \%$ on microbiological diagnostics, $11 \%$ on isolation, $10 \%$ on extra personnel, $5 \%$ on cleaning and $7 \%$ on other expenditures) [13]. Part of these savings are direct (e.g., diagnostics, extra personnel) and part are indirect such as preventing the closure of beds or opportunity costs due to redistributing personnel, which in turn can lead to an increase in revenue and improved quality of care. In both studies, infection rates were low and most patients were only colonized, making the impact on medication costs small. Included parameters are however not a complete overview of all costs and benefits. Considering the numerous other positive effects of an infection prevention department on nosocomial infections, other small nonoutbreak situations and antimicrobial resistance rates, potential benefits might be substantially higher. Furthermore, expected benefits from a societal perspective are even bigger, especially if looking to the increasing problems of

\section{EXECUTIVE SUMMARY}

- Patients colonized with (resistant) pathogenic microorganisms are a threat for other patients in healthcare centers, as they can lead to severe infections.

- Multi-drug-resistant organisms are a problem for healthcare workers as they can become colonized, spread it to other patients and, depending on the national guidelines, being a problem to employment in healthcare settings.

- It is therefore important for healthcare centers to have an effective, institution-wide infection prevention and control program.

- Our academic hospital saw a continuous rise in (mostly admitted) colonized patients with risk microorganisms over the last 8 years (2007-2014).

- During these 8 years, more money was spent on infection prevention and control each year, leading to more measures done each year as well.

- The efforts of the implementation of infection control can be measured in standardized and comparable way.

- The number of outbreak patients in the same 8 years was less than the predicted number suggesting a preventive effect of the extra infection prevention and control money.

- Patients were protected from colonization of multi-drug-resistant organisms and subsequent infections.

- These prevented cases saved enough money each year, to earn back the infection prevention and control investments, making them highly cost efficient. 
antimicrobial resistance. Taken together, it is a bundle of capable and sufficient infection prevention personnel, and proper protocols that are correctly followed. By doing so, the hospital could prevent outbreak patients thereby improving patient safety. In conclusion, infection prevention will usually save a sufficient amount of resources to become highly cost beneficial.

\section{Acknowledgements}

The authors would like to thank A Hake and J Oosterhof from the purchasing department of the University Medical Center Groningen in their help to obtain the product consumption data. The authors are also grateful for the support and fruitful discussions with had with J van der Weerd, manager of the Infection Prevention Unit of the University Medical Center Groningen, $R$ Hendrix of Certe Laboratories Groningen and H Grundmann of the University Medical Center Groningen and the University Hospital Freiburg, Germany.

\section{Financial \& competing interests disclosure} This work was partly supported by the EU, the German states of North Rhine-Westphalia and Lower Saxony and the Dutch provinces Overijssel, Gelderland and Limburg via the EurSafety Health-net project [Interreg IVa III-1-01=073]. $B$ Sinha has received a travel grant co-funded by Pfizerl Wyeth, and worked on projects in cooperation with Pathogenica, Life Technologies and Copan. The authors have no other relevant affiliations or financial involvement with any organization or entity with a financial interest in or financial conflict with the subject matter or materials discussed in the manuscript apart from those disclosed.

No writing assistance was utilized in the production of this manuscript.

\section{Open access}

This work is licensed under the Creative Commons Attribution-NonCommercial 4.0 Unported License. To view a copy of this license, visit http://creativecommons.org/ licenses/by-nc-nd/4.0/

\section{References}

1 Eber MR, Laxminarayan R, Perencevich E, Malani A. Clinical and economic outcomes attributable to health care-associated sepsis and pneumonia. Arch. Intern. Med. 170 (4), 347-353 (2010).

2 Klevens RM, Edwards JR, Richards CL Jr Estimating health care-associated infections and deaths in U.S. hospitals, 2002. Public Health Rep. 122(2), 160-166 (2007).

3 Plowman R, Graves N, Griffin MA et al. The rate and cost of hospital-acquired infections occurring in patients admitted to selected specialties of a district general hospital in England and the national burden imposed. J. Hosp. Infect. 47(3), 198-209 (2001).

4 Graves N. Economics and preventing hospital-acquired infection. Emerg. Infect. Dis. 10(4), 561-566 (2004).

5 Bode LG, Wertheim HF, Kluytmans JA et al. Sustained low prevalence of meticillinresistant Staphylococcus aureus upon admission to hospital in The Netherlands. J. Hosp. Infect. 79(3), 198-201 (2011).

6 Grayson ML, Russo PL, Cruickshank M et al. Outcomes from the first 2 years of the Australian National Hand Hygiene Initiative. Med. J. Aust. 195(10), 615-619 (2011).

7 Stone SP, Fuller C, Savage J et al. Evaluation of the national Cleanyourhands campaign to reduce Staphylococcus aureus bacteraemia and Clostridium difficile infection in hospitals in England and Wales by improved hand hygiene: four year, prospective, ecological, interrupted time series study. BMJ 344, e3005 (2012).

8 Wolkewitz M, Barnett A, Palomar Martinez M, Frank U, Schumacher M. IMPLEMENT Study Group. Interventions to control nosocomial infections: study designs and statistical issues. J. Hosp. Infect. 86(2), 77-82 (2014).

9 Graves N. How costs change with infection prevention efforts. Curr. Opin. Infect. Dis. 27(4), 390-393 (2014).

10 Stone PW, Larson E, Kawar L. A systematic audit of economic evidence linking nosocomial infections and infection control interventions: 1990-2000. Am. J. Infect. Control. 30(3), 145-152 (2002).

11 Werkgroep Infectie Preventie (WIP). www.wip.nl

12 DESAN. www.desan.nl

13 Dik JH, Dinkelacker AG, Vemer P et al. Costanalysis of seven nosocomial outbreaks in an academic hospital. PLoS ONE 11(2), e0149226 (2016).

14 WIP. [MRSA guideline Hospitals]. WIP website. www.rivm.nl

15 European Centre for Disease Prevention and Control. Antimicrobial resistance surveillance in Europe 2012. Annual Report of the European Antimicrobial Resistance Surveillance Network (EARS-Net). TQ-AM-13-001-EN-CECDC; Stockholm, Sweden (2013).

http://ecdc.europa.eu
16 Donker T, Wallinga J, Grundmann H. Patient referral patterns and the spread of hospital-acquired infections through national health care networks. PLoS Comput. Biol. 6(3), e1000715 (2010).

17 van der Bij AK, Kardamanidis K, Frakking FN, Bonten MJ. Signaleringsoverleg Ziekenhuisinfecties en Antimicrobiële Resistentie. [Nosocomial outbreaks and resistant microorganisms]. Ned. Tijdschr. Geneeskd. 159, A8585 (2015).

18 Dik JH, Poelman R, Friedrich AW et al. An integrated stewardship model: antimicrobial, infection prevention and diagnostic (AID). Future Microbiol. 11(1), 93-102 (2016).

19 Zingg W, Mutters NT, Harbarth S, Friedrich AW. Education in infection control: A need for European certification. Clin. Microbiol. Infect. 21(12), 1052-1056 (2015).

20 VHIG. [Norm infection prevention specialists]. VHIG website. www.vhig.nl

21 van den Broek PJ, Kluytmans JA, Ummels LC, Voss A, Vandenbroucke-Grauls CM. How many infection control staff do we need in hospitals? J. Hosp. Infect. 65(2), 108-111 (2007).

22 Mitchell BG, Hall L, MacBeth D, Gardner A, Halton K. Hospital infection control units: Staffing, costs, and priorities. Am. J. Infect. Control. 43(6), 612-616 (2015).

23 Stone PW, Pogorzelska-Maziarz M, Herzig CT et al. State of infection prevention in US hospitals enrolled in the National Health and 
Safety Network. Am. J. Infect. Control. 42(2), 94-99 (2014).

24 Spijkerman I, Ruijs G, Kluytmans JA. [The future of infection prevention. Part 1 guidelines and implementation.]. Ned. Tijdschr. Med. Microbiol. 20(2), 70-72 (2012).

25 Spijkerman I, Ruijs G, Kluytmans JA. [The future of infection prevention. Part 2 Norm, collaboration, education and funding.]. Ned. Tijdschr. Med. Microbiol. 20(4), 149-152 (2012).

26 NVMM. [Norm infection prevention specialists 2012]. NVMM website. www.nvmm.nl
27 Hugonnet S, Harbarth S, Sax H, Duncan RA, Pittet D. Nursing resources: a major determinant of nosocomial infection? Curr. Opin. Infect. Dis. 17(4), 329-333 (2004).

28 Stone PW, Mooney-Kane C, Larson EL et al. Nurse working conditions and patient safety outcomes. Med. Care 45(6), 571-578 (2007).

29 Rogowski JA, Staiger D, Patrick T, Horbar J, Kenny M, Lake ET. Nurse staffing and NICU infection rates. JAMA Pediatr. 167(5), 444-450 (2013).

30 Dutch Hospital Data. www.dhd.nl
31 Chakravarthy M, Adhikary R, Gokul B, Pushparaj L. Hospital acquired infection is inversely related to utilization of isopropyl alcohol and tissue paper pulls-a prospective observational study. J. Assoc. Physicians India 59, 548-550 (2011).

32 Bittner MJ, Rich EC. Surveillance of handwashing episodes in adult intensivecare units by measuring an index of soap and paper towel consumption. Clin. Perform. Qual. Health Care 6(4), 179-182 (1998). 